\title{
Co-Morbidity and COVID-19: A Study of Case Severity and Early Outcome
}

\author{
Ankita Goyal ${ }^{1}$ (D), Nitin Tiwari ${ }^{1}$ (D), Rohit Patawa ${ }^{2 *}$ (D)
}

${ }^{1}$ Autonomous State Medical College, Community Medicine, Firozabad, India

${ }^{2}$ University of Allahabad, Department of Statistics, Uttar Pradesh, India

\section{Correspondence: \\ Rohit Patawa}

Address: Department of Statistics, University of Allahabad, Uttar Pradesh, India

Email: rohitpatawa@gmail.com

Received: 07.12.2021,

Accepted: 16.01.2022

https://doi.org/10.29333/jcei/11583

\begin{abstract}
Background: The corona virus disease 2019 (COVID-19) has spread around the world and mutating rapidly. It is absolutely essential to evaluate the potential risk factor influencing disease progression and better understanding of high risk co-morbidities among COVID-19 patients.
\end{abstract}

Objectives: To evaluate the association of serious events in COVID-19 patients according to the number and type of co-morbidities also the association of co-morbidities with the adverse events and the risk of early outcomes with any co-morbidity.

Methods: This was a prospective observational study among 1,583 laboratory-confirmed COVID-19 cases admitted during the study period from Apr 2020 to March 2021. Odds ratio $(\mathrm{OR})$ and risk ratio $(\mathrm{RR})$ with respective confidence intervals have been calculated to evaluate the presence and strength of association of case severity with the presence and number of co morbidities.

Results: There was 302 study subjects with one or more than one co-morbidities having the mean (SD) age of 54(15) year and 206(68.2\%) male cases compared to female. The leading comorbidities were diabetes $(146,9.2 \%)$, hypertension $(142,9 \%)$ with respiratory disease $(50$, $3.2 \%)$, CAD $(16,1 \%)$. Any co-morbid patient has 6 times odds of getting admitted in ICU in comparison to non-co-morbid patient while the risk ratio signifies that five times higher chance of getting admitted in ICU than any non-co-morbid patient. The chances of death in them are always higher than non-co-morbid specially diabetes and hypertensive with CAD.

Conclusions: Among patients with COVID-19 disease any co-morbidity resulted to poorer clinical outcomes and more the number of co-morbidities, poorer the clinical outcomes.

Keywords: COVID-19, co-morbidity, diabetes, odds ratio, risk ratio

\section{INTRODUCTION}

Since December 2019, a novel coronavirus disease (COVID-19), caused by severe acute respiratory syndrome corona-virus 2 (SARS-CoV-2), has scourged the world, resulting in the WHO declaring it as a pandemic. As of November 26, 2021, COVID-19 has affected more than 259 million people in 220 countries around the world, with the majority of cases being reported from India followed by the United States. The absolute number of deaths has already surpassed 5.1 million globally and is expected to increase further as the virus is mutating. COVID-19 has contributed to an enormous adverse impact globally and become a public health emergency of international concern [1]. The disease also infiltrated the Indian masses and has reached 34.5 million laboratory-confirmed cases and around 0.47 million deaths as of November 26, 2021[2].

Severe acute respiratory syndrome coronavirus 2 (SARS-CoV-2) affects people of all age groups, but the risk is high in older age group and people with preexisting comorbidities which include cardiovascular diseases, hypertension, chronic respiratory disease, chronic obstructive pulmonary disease (COPD), diabetes, thyroid, and cancer [3-5], it is also observed that these comorbidities increase the chance of infection [6]. Patients with hypertension and diabetes have shown worse prognosis and have increased morbidity and mortality rate with higher number and chances of ICU 
admission during hospitalization [7]. The previous coexisting illness was seen in severe disease group in 39\% patients as compared to $21 \%$ patients in non-severe disease group of COVID-19 [8].

For the prevalence of co-morbidities, several studies and meta-analysis have been conducted worldwide. In a metaanalysis of 1,786 patients, hypertension (15.8\%) was found most common co-morbidity preceding with cardiovascular and cerebrovascular conditions (11.7\%) and diabetes (9.4\%) $[6,9]$. On admission, $20-51 \%$ of patients reported as having at least one co-morbidity, with diabetes (10-20\%), hypertension (10-15\%) cardiovascular and cerebrovascular diseases (7-40\%) as being most common [10,11]. According to a study on Chinese patients' hypertension (19\%) was most common co-morbidity followed by diabetes (9\%) among COVID-19 patients [12]. In almost all the studies concerned with COVID-19, HIV, hepatitis B, malignancy, respiratory illnesses, renal disorders, and immune deficiencies were identified as the less common co-morbidities among COVID-19 patients.

In the study of outcome of co-morbid COVID-19 patients, diabetes was identified most importance cause of mortality in hospitalized patients according to a systematic review and meta-analysis [13]. Similar conclusion has also been found in the study of 395 young adults of New York City that prevalence of mortality among diabetic patients was around 74\% during hospitalization [14]. A systematic review and meta-analysis was done to evaluate the impact of various co-morbidities on serious events identified. The occurrences of serious events were $13.16 \%$ among the hypertensive and diabetic patients and also, the other co-morbidities such as cardio vascular disease, COPD and chronic kidney disease had significant association with serious events [15].

During this pandemic a better understanding of highrisk co-morbidities may be helpful to identify high risk patients during admission to health facility. Serious events include: ICU admission, novel corona virus pneumonia (NCP), mechanical ventilation, ARDS, and death. With the rapid spread of the virus causing COVID-19 disease and because of lack of adequate health-care infrastructure and human resources there is a need to formulate a treatment strategy for various co-morbidities leading to disabilities and death. Our objective was to evaluate the association of serious events in COVID-19 patients according to the number and type of co-morbidities. Also, the association of co-morbidity with adverse events and risk of early outcomes has been evaluated.

\section{MATERIALS AND METHODS}

A prospective observational study was conducted among patients admitted with COVID-19 infection at tertiary care hospital in Firozabad City of Uttar Pradesh dedicated for COVID-19 patients. Total 1,583 laboratory-confirmed COVID-19 cases were admitted during the study period. Out of which, a cohort of 302 patients was found with one or more than one co-morbidity based on patient's self-report and after clinical evaluation on admission. These patients were followed for their clinical progress and adverse events on daily basis during their stay in hospital. Co-morbidities were initially treated as a categorical variable (Yes vs. No), and subsequently classified on the basis number (Single vs. Multiple). A structured format has been designed for day-today progress of these subjects and entered into a computerized database by team of trained data analyst for further confirmation. The primary end point was a composite measure which consisted of the admission to high dependency unit, or intensive care unit (ICU), or invasive ventilation or referral to higher center.

Statistical analyses were conducted with SPSS software version 23.0 (Chicago, IL, USA). This is time-based sample analysis hence no sample size calculation has been made but the prevalence of co-morbidities is same as the national-wide figure hence it is representative of national patient's figure. Also, the sample size was sufficient to power of statistical analyses. The continuous variables are presented as means and the qualitative variables are presented as counts as well as in percentages.

The Chi-square test has been used to test the statistical significance of association between different adverse events and type of co-morbidities respectively. Then after the odds ratios (OR) have been calculated for all the adverse events with their respective 95\% confidence intervals (CIs) to obtain the strength of association between co-morbidities and adverse events. The risk ratios or relative risks (RR) have also been calculated with their respective 95\% confidence intervals to discuss the relative risk associated with comorbid patients for serious events.

\section{RESULTS}

\section{Demographic and Co-Morbid Characteristics}

Among 302 study subjects with one or more than one comorbidity, the mean (SD) age of co-morbid patient group was 54(15) and 206(68.2\%) male subjects. The prevalence of the specific co-morbidities was diabetes (146, 9.2\%), hypertension $(142,9 \%)$, respiratory disease $(50,3.2 \%)$, CAD $(16,1 \%)$, malignancy $(8,0.5 \%)$, thyroid $(40,2.5 \%)$, and others $(29,1.8 \%)$. Most of the patients suffering from any specific co-morbidity were male except in the case of thyroid. The mean of these co-morbid groups was above 50 years while the patients suffering from hypertension or CAD were with the mean (SD) age of 61-year (14) (Table 1). 198 subjects have single co-morbidity while $104(34.4 \%)$ patients were reported with two or more co-morbidities. The study patients with two or more than two co-morbidities were older than the patient with one co-morbidity (Mean age (SD): 58-year (15) vs 52-year (15)) (Table 2). Among these co-morbid patients, $88(29.1 \%)$ has been admitted to ICU in which $29(9.6 \%)$ were on either mechanical ventilation or 
Co-morbidity and COVID-19

Table 1. Demographic Characteristics and Prevalence of Co-Morbidities Among Study Subjects

\begin{tabular}{lccccc}
\hline Co-Morbidity & $\mathbf{N = 3 0 2}$ & Male & Female & Mean (SD) Age & Prevalence \\
\hline DM (Diabetes Mellitus) & 146 & $99(68 \%)$ & $47(32 \%)$ & $55(12)$ & $9.2 \%$ \\
\hline HTN (Hypertension) & 142 & $89(63 \%)$ & $43(37 \%)$ & $59(14)$ & $9.0 \%$ \\
\hline RD (Respiratory Disease) & 50 & $35(70 \%)$ & $15(30 \%)$ & $50(17)$ & $3.2 \%$ \\
\hline CAD (Coronary Artery Disease) & 16 & $13(81 \%)$ & $3(19 \%)$ & $61(14)$ & $1.0 \%$ \\
\hline TD (Thyroid Disease) & 40 & $11(28 \%)$ & $29(73 \%)$ & $51(16)$ & $2.5 \%$ \\
\hline Cancer & 8 & $6(75 \%)$ & $2(25 \%)$ & $56(09)$ & $0.5 \%$ \\
\hline Other & 29 & $23(79 \%)$ & $6(21 \%)$ & $46(17)$ & $1.8 \%$ \\
\hline
\end{tabular}

Table 2. Demographic Characteristics and Numbers of Co-Morbidities Among Study Subjects

\begin{tabular}{lccccc}
\hline Number of Co-Morbidity & $\mathbf{N = 3 0 2}$ & Male & Female & Mean (SD) Age & Prevalence \\
\hline One & 198 & $143(73 \%)$ & $55(27 \%)$ & $52(15)$ & $12.5 \%$ \\
\hline Two or More & 104 & $61(59 \%)$ & $43(41 \%)$ & $58(15)$ & $6.6 \%$
\end{tabular}

Table 3. Association Between Presence of Co-Morbidity and Serious Events Study Subjects

\begin{tabular}{lcccc}
\hline Events & Calculated Chi-square & p-value & OR \& (Cl) & RR \& (CI) \\
\hline ICU admission & 143.5 & $<0.05(0.2 \mathrm{e}-15)$ & $6.6(9.3-4.7)$ & $5.0(6.6-3.8)$ \\
\hline Adverse Event & 52.1 & $<0.05(0.53 \mathrm{e}-11)$ & $7.1(12.8-3.9)$ & $6.5(11.4-3.7)$ \\
\hline Death & 44.8 & $<0.05(0.22 \mathrm{e}-9)$ & $17.9(53.8-5.9)$ & $17.0(50.4-5.7)$ \\
\hline
\end{tabular}

referred to higher center for further interventions. Out of the co-morbid patients, 17(5.4\%) deaths have been reported.

\section{Association Between Presence of Co-Morbidity and}

\section{Serious Events}

In this analysis, ICU admission, adverse events and death were considered as serious events. To determine the association of co-morbidity with all the serious events, chi square test has been used. The presence of co-morbidity has high degree of association with the occurrence of serious events as all the p-values are less than 0.05 . The odds ratio (OR) of ICU admission between co-morbid patients and non-co-morbid patients was 6.6 with confidence interval (4.7 to 9.3) while the risk ratio (RR) was 5.0 with CI (3.8 to 6.6). it indicates that any co-morbid patient has 6 times odds of getting admitted in ICU with respect to any non-co-morbid patient while the risk ratio signifies that any co-morbid patient has 5 times higher chance of getting admitted in ICU than any non-co-morbid patient. Similarly, the odds ratio of adverse events between co-morbid and non-co-morbid patients was 7.1 with CI (3.9 to 12.8) describes that the comorbid patient has 7 times odds of having any adverse event than non-co-morbid patient. The value of the risk ratio (RR) was 6.5 with CI (3.7 to 11.4) indicates that chances of any adverse event with co-morbid patient has 6.5 times higher than any non-co-morbid patient (Table 3).

When considering death, the odds ratio was 17.9 with CI ranging 5.9 to 53.8 between co-morbid and non-co-morbid patient and the RR was 17.0 with $\mathrm{CI}$ ranging 5.7 to 50.4 which shows the higher odds as well as chances of death of comorbid patients in comparison of any non-co-morbid patient. The range of confidence intervals of OR and RR for death were measured too high (about 44) which indicates skewness in the chances of death of co-morbid patients although lower limits of CIs for both the measures (OR \& $\mathrm{RR})$ are positive $(>1)$ which clue towards the chances of death in co-morbid patients are always higher than non-comorbid patients (Table 3).

\section{Association Between Different Types of Co- Morbidities with Serious Events}

In this analysis, the measure of association between the type of co-morbidities with different serious events was considered. Table $\mathbf{4}$ has summarized the statistical significance measure between the type of co-morbidities with serious events. Odds ratio of ICU admission between the patient with diabetes and non-diabetes was 12.9 with the 95\% CI ranging from 8.1 to 20.5 and the RR was 9.4 with $\mathrm{CI}$ ranging from 6.4 to 13.8 . This indicates higher odds and chances of diabetic patients to get admitted in ICU. This association is statistically significant as its p-value (2.20E-16) is less than 0.05 . Similar results can be seen as the odds ratio of adverse events between COVID-19 patients with diabetes and without diabetes was 4.4 with $\mathrm{CI}$ ranging from 2.3 to 8.4 and the RR was 4.1 with $\mathrm{CI}$ ranging from 2.2 to 7.4 and this association is also statistically significant as p-value (4.30E$06(<0.05))$. In the case of death, the association is still significant (as p-value is $1.68 \mathrm{E}-07$ less than 0.05 ) and large in strength as the odds ratio was 10.4 and the RR was 9.8 but the larger width of CI of both measures (OR \& RR) indicates the larger variability in the chances and odds of death of COVID-19 patients with diabetes as compared to nondiabetic COVID-19 patients.

The association of hypertension as co-morbidity with any type of serious event was statistically significant as all the pvalues were less than 0.05 . The odds ratio of ICU admission between hypertensive patients and non-hypertensive patients was 11.4 with CI ranging from 7.1 to 18.1 and the 
Co-morbidity and COVID-19

Table 4. Association Between Different Types of Co-Morbidities with Serious Events in Study Subjects

\begin{tabular}{|c|c|c|c|c|c|c|c|c|c|}
\hline \multirow{3}{*}{$\begin{array}{l}\text { Type of Co- } \\
\text { Morbidity }\end{array}$} & \multicolumn{9}{|c|}{ Serious Events } \\
\hline & \multicolumn{3}{|c|}{ ICU Admission } & \multicolumn{3}{|c|}{ Adverse Events } & \multicolumn{3}{|c|}{ Death } \\
\hline & p-value & OR \& (CI) & $\mathrm{RR} \&(\mathrm{Cl})$ & p-value & OR \& (Cl) & RR \& (Cl) & p-value & OR \& (CI) & RR \& (CI) \\
\hline Diabetes & $<0.05$ & $12.9(8.1-20.5)$ & $9.4(6.4-13.8)$ & $<0.05$ & $4.4(2.3-8.4)$ & $4.1(2.2-7.4)$ & $<0.05$ & $10.4(3.8-28.0)$ & $9.8(3.7-25.8)$ \\
\hline Hypertension & $<0.05$ & $11.4(7.1-18.1)$ & $8.5(5.8-12.4)$ & $<0.05$ & $7.7(3.6-16.5)$ & 7.2(3.5-14.7) & $<0.05$ & $4.7(1.6-13.9)$ & $4.6(1.6-13.1)$ \\
\hline $\begin{array}{l}\text { Respiratory } \\
\text { Disease }\end{array}$ & $<0.05$ & $13.0(7.0-24.2)$ & $8.4(5.5-12.9)$ & $<0.05$ & $9.0(3.5-23.1)$ & $8.0(3.4-18.8)$ & $<0.05$ & $11.0(3.4-35.5)$ & $10.2(3.4-30.6)$ \\
\hline CAD & $<0.05$ & $14.3(5.0-40.6)$ & $9.3(4.7-18.4)$ & 0.02374 & $8.1(1.8-37.6)$ & $7.3(1.9-28.0)$ & 0.3954 & $6.9(0.9-55.6)$ & $6.5(0.9-46.5)$ \\
\hline Thyroid & $<0.05$ & $6.3(3.0-13.3)$ & $4.9(2.8-8.8)$ & 0.3723 & $1.9(0.7-5.5)$ & $1.8(0.7-4.8)$ & 0.8782 & $2.6(0.3-20.3)$ & $2.6(0.3-19.0)$ \\
\hline Cancer & 0.03638 & $8.0(1.6-40.4)$ & $6.3(1.8-21.3)$ & 0.3502 & $7.9(0.9-66.3)$ & $7.0(1.1-45.6)$ & - & - & - \\
\hline
\end{tabular}

chance of hypertensive patient getting admitted in ICU was 8.5 times higher than non-hypertensive patients with COVID-19 as the RR was 8.5 having CI from 5.8 to 12.4 . The odds ratio of adverse events for similar group was 7.7 (with $95 \%$ CI from 3.6 to 16.5 ) and RR was 7.2 with CI ranging from 3.5 to 14.7 which indicates the higher chance of adverse events for COVID-19 patient with hypertension in comparison of non-hypertensive patient. The odds ratio and $\mathrm{RR}$ also signifies the higher odds and chance of death in COVID-19 patients with hypertension having the odds ratio 4.7 and RR 4.6.

The odds ratio between COVID-19 patients with CAD and without CAD was 14.3 for ICU admission with CI ranging from 5.0 to 40.6 and the p-value was less than 0.05 . Similarly, for adverse events, odds ratio was 8.1 with CI 1.8 to 37.6 and p-value less than 0.05 while for death the odds ratio was 6.9 with $\mathrm{CI}$ ranging from 0.9 to 55.6 with p-value 0.395. This shows that the COVID-19 patients with CAD have higher odds of getting admitted in ICU, adverse events and death in comparison of COVID-19 patients without CAD but the association with death was not statistically significant also its confidence intervals had large width and lower value (0.9) is less than one. Also, from the values of risk ratios of serious events between same group of patients (with CAD and without CAD), it can be observed that CAD patients have 9 times higher chances of getting admitted in ICU and around 8 times higher experience of adverse event during hospitalization while around 6 times greater chance of death although it was not statistically significant.

The p-values for an association between respiratory diseases as co-morbidity and serious events show the statistical significance. An odds ratio of ICU admission between patients with respiratory disease and patients without respiratory disease was 13.0 with CI ranging from 7 to 24.2 and the odds ratio of adverse events was 9.0 with CI ranging from 3.5 to 23.1 while the odds ratio of death was 11.0 with CI from 3.4 to 35.5 . Odds ratio between the patients with any respiratory disease and patients without respiratory disease suggests that any COVID-19 patients with respiratory disease has higher odds of experiencing any type of serious event in the comparison of COVID-19 patients without respiratory disease. The values of risk ratios (RR) among these is greater than one for any type of serious events and both the limits (lower \& upper) 95\% CI are greater than one. This shows that the COVID-19 patients with respiratory disease have higher risk of serious events than COVID-19 patients without respiratory disease. From the values of RR, it can be seen that COVID-19 patients with respiratory disease have eight times higher chance of ICU admission and getting any adverse events while ten times higher chances of death as compared to COVID-19 patients without respiratory disease.

The association between ICU admission and thyroid abnormalities was statistically significant with odds ratio 6.3 having CI ranging from 3.0 to 13.3 and RR was 2.8 with CI ranging from 1.8 to 8.8 , which shows higher odds and chance of getting admitted in ICU in comparison of patients without thyroid abnormalities. The odds ratios and RRs for rest of comorbidities were greater than one but highly insignificant as their p-values for association were greater than 0.05 (Table 4).

\section{DISCUSSION}

This study has been undertaken to find out the association of serious events in COVID-19 patients according to the number and type of co-morbidities and also to determine the risk of adverse events in co-morbid COVID-19 patients. In our study, around 19.1\% study subjects reported with at least one co-morbidity whereas 20$51 \%$ of patients having at least one co-morbidity in the study conducted by $[10,11]$ while in the study of [8], $8.2 \%$ patients reported having two or more co-morbidities. The finding of our study showed that the most prevalent co-morbidity was diabetes $(9.2 \%)$ followed by hypertension (9\%). which is similar to the finding of studies in Wuhan, China with diabetes (10-20\%) and hypertension (10-15\%). According to the study by [8], the hypertension (16.9\%) was most prevalent co-morbidity followed by diabetes (8.2\%).

The co-morbid patient has 6-times higher odds of getting admitted in ICU and 5 times higher chance of getting admitted in ICU than any non-co-morbid patient. Similarly, the co-morbid patient has 7 times higher odds of having any adverse event than non-co-morbid patient. Similar finding was seen in [16], which shown hypertension, diabetes, COPD, cardiovascular disease, and cerebrovascular disease as major risk factors for patients with COVID-19. 
When considering an association between types of comorbidities with serious events, it has been found that there are higher chances of ICU admission and occurrence of adverse events among co-morbid patients and most common among patients with diabetes, hypertension, and respiratory diseases. According to [15], the presence of hypertension with diabetes mellitus, and COPD had significant association in patients with COVID-19 on having serious events. The co-morbidities had a major effect on patients with COVID-19 and leads to higher chances of serious events, diabetic patients had 3.07-fold higher chances of severe events which were statistically significant ( $p$ value $<0.001)$. The presence of diabetes has statistically significant impact on death in COVID-19 patients. Presence of COPD had a 6.6 times higher risk of developing serious events in COVID-19 patients.

The chances of death of co-morbid patients have positive lower limits of CIs for both the measures (OR \& RR), which concludes towards the chances of death in co-morbid patients are always higher than non-co-morbid patients. According to the [8], the percentage of deaths observed in COVID-19 patients with co-morbidity such as cardiovascular disease (13.2\%) and diabetes (9.2\%), chronic respiratory disease $(8 \%)$, hypertension $(8.4 \%)$, and cancer (7.6\%) are higher as compared to patients without coexisting conditions. The risk of rapid spread of disease leads to higher chances of morbidity and mortality amongst the patients with co-morbidities. COVID-19 disease has placed a significant burden on the health care system of countries mainly with limited resources including personal protection equipment, medications, and ventilators [17-19].

There were some limitations of the study. Underreporting of co-morbidities leads to underestimation of the true strength of association with the clinical prognosis. It could also lead to over-estimation of strength of association with adverse outcome. The duration of follow-up was relatively short so the studies with long-time frame are need of hour for exploring the association's case severity and early outcome. The limited number of enrolled patients leads to smaller sample size for analysis. We believe that these differences could have changed the outcomes. Therefore, studies that include an external validation of the results would be desirable. Finally, because COVID-19 spread almost all over the world so it is necessary to analyze stratified subgroup by the specific co-morbidities and their association with the risk of death.

\section{CONCLUSIONS}

The findings suggested that COVID-19 patients with comorbidities had greater disease severity and having high risk of developing serious events i.e., ICU admission, mechanical intubation and death. So, the analysis of association of comorbidities with severe events in COVID-19 can help policy makers and physicians focus more on these high-risk patients. A universal protocol and guidelines can be created for patients with co-morbidity and COVID-19. Diabetes mellitus and respiratory diseases are the co-morbidity having a significant impact on death in COVID-19 patients. The proper triage of these patients should be implemented because this will help identify those patients who would be more likely to develop serious events. Knowledge of these comorbidities can help us better stratify COVID-19 patients at higher risk allowing a more targeted and specific approach in preventing fatal events. Prompt intervention and focused care should be given to the patients with co-morbidities upon COVID-19 confirmation.

Author contributions: All authors have sufficiently contributed to the study, and agreed with the results and conclusions.

Funding: No funding source is reported for this study.

Declaration of interest: No conflict of interest is declared by authors.

\section{REFERENCES}

1. WHO. Available at: https://www.who.int

2. MOHFW. Available at: https://www.mohfw.gov.in

3. Zhou F, Yu T, Du R, et al. Clinical course and risk factors for mortality of adult inpatients with COVID-19 in Wuhan, China: A retrospective cohort study. Lancet. 2020;395(10229):1054-62. doi: 10.1016/S01406736(20)30566-3.

4. Sanyaolu A, Okorie C, Marinkovic A, et al. Comorbidity and its impact on patients with COVID-19. SN Compr Clin Med. 2020;1-8. doi: 10.1007/s42399-020-00363-4.

5. Zhou Y, Yang Q, Chi J, et al. Comorbidities and the risk of severe or fatal outcomes associated with coronavirus disease 2019: A systematic review and meta-analysis. Int J Infect Dis. 2020;99:47-56. doi: 10.1016/j.ijid.2020.07. 029.

6. CDC. Coronavirus (COVID-19): Symptoms of coronavirus. Centers for Disease Control and Prevention. 2021. Available at: https://www.cdc.gov/ coronavirus/2019-ncov/symptomstesting/symptoms. html

7. Singh AK, Gupta R, Ghosh A, Misra A. Diabetes in COVID-19: Prevalence, pathophysiology, prognosis and practical considerations. Diabetes Metab Syndr. 2020;14(4):303-10. doi: 10.1016/j.dsx.2020.04.004.

8. Guan WJ, Ni ZY, Hu Y, et al. Clinical characteristics of coronavirus disease 2019 in China. N Engl J Med. 2020;382(18):1708-20. doi: 10.1056/NEJMoa2002032.

9. Paudel SS. A meta-analysis of 2019 novel coronavirus patient clinical characteristics and comorbidities. Res Sq. 2020. doi: 10.21203/rs.3.rs-21831/v1.

10. Huang C, Wang Y, Li X, et al. Clinical features of patients infected with 2019 novel coronavirus in Wuhan, China. Lancet. 2020;395(10223):497-506. doi: 10.1016/S01406736(20)30183-5. 
11. Chen N, Zhou M, Dong X, et al. Epidemiological and clinical characteristics of 99 cases of 2019 novel coronavirus pneumonia in Wuhan, China: A descriptive study. Lancet. 2020;395(10223), 507-513. doi: 10.1016/S0140-6736(20)30211-7.

12. Yin T, Li Y, Ying Y, Luo Z. Prevalence of comorbidity in Chinese patients with COVID-19: Systematic review and meta-analysis of risk factors. BMC Infect Dis. 2021;21(1):200. doi: 10.1186/s12879-021-05915-0.

13. Corona G, Pizzocaro A, Vena W, et al. Diabetes is most important cause for mortality in COVID-19 hospitalized patients: Systematic review and meta-analysis. Rev Endocr Metab Disord. 2021;22(2):275-96. doi: 10.1007/s11154-021-09630-8.

14. Altonen BL, Arreglado TM, Leroux O, MurrayRamcharan M, Engdahl R. Characteristics, comorbidities and survival analysis of young adults hospitalized with COVID-19 in New York City. PloS One. 2020;15(12):e0243343. doi: 10.1371/journal.pone. 0243343 .
15. Nandy K, Salunke A, Pathak SK, et al. Coronavirus disease (COVID-19): A systematic review and metaanalysis to evaluate the impact of various comorbidities on serious events. Diabetes Metab Syndr. 2020;14(5):1017-25. doi: 10.1016/j.dsx.2020.06. 064.

16. Wang B, Li R, Lu Z, Huang Y. Does comorbidity increase the risk of patients with COVID-19: Evidence from metaanalysis. Aging (Albany NY). 2020;12(7):6049-57. doi: 10.18632/aging.103000.

17. Yu J, Ouyang W, Chua MLK, Xie C. SARS-CoV-2 transmission in cancer patients of a tertiary hospital in Wuhan. JAMA Oncol. 2020;6(7):1108-10. doi: 10.1001/ jamaoncol.2020.0980.

18. Lu H, Stratton CW, Tang Y-W. Outbreak of pneumonia of unknown etiology in Wuhan, China: The mystery and the miracle. J Med Virol. 2020;92(4):401-2. doi: 10.1002/jmv.25678.

19. Burki TK. Cancer guidelines during the COVID-19 pandemic. Lancet Oncol. 2020;21(5):629-30. doi: 10.1016/S1470-2045(20)30217-5. 